\title{
Modelling of top quark pair production in association with a Standard Model boson or a heavy quark pair
}

\author{
María Moreno Llácer ${ }^{* \dagger}$ \\ II. Physikalisches Institut, Georg-August-Universität Göttingen \\ E-mail: mamolla@cern.ch
}

\begin{abstract}
The production of top quark pairs in association with heavy Standard Model bosons or with heavy flavour quark pairs is important both as a signal and a background in several ATLAS analyses. At present, strong constraints on such processes cannot be obtained from data. Therefore, their modelling using Monte Carlo simulations as well as the associated uncertainties are of critical importance. This work documents the Monte Carlo samples currently being used in ATLAS for $t \bar{t} H, t \bar{t} V$ ( $V=W, Z$ vector bosons) and $t \bar{t}+$ bottom quark pair processes for $\sqrt{s}=13 \mathrm{TeV}$ proton-proton collisions.
\end{abstract}

38th International Conference on High Energy Physics

3-10 August 2016

Chicago, USA

* Speaker.

$\dagger$ on behalf of the ATLAS Collaboration 


\section{Why $t \bar{t}+X$ Monte Carlo studies?}

The productions of a Higgs or vector boson $(W, Z)$ in association with a top quark pair (referred to as $t \bar{t} H, t \bar{t} W$ and $t \bar{t} Z$ ) are some of the main highlights of the physics program of the LHC Run 2. The large center-of-mass energy available allows for the copious production of top quarks pairs in association with other particles at high transverse momentum enabling precise Standard Model (SM) measurements as well as probing many Beyond SM models that enhance rates for $t \bar{t}+X$ production. Precise measurements of the production cross sections require an excellent understanding and modelling of QCD effects. Recently, the emphasis has been on identifying and characterizing state-of-the-art theoretical predictions and tools for signals and backgrounds in all relevant $t \bar{t} H, t \bar{t} W$ and $t \bar{t} Z$ searches, as well as on identifying various theory-related sources of uncertainties. The latter are becoming the dominant uncertainties in these searches (e.g. [1]) and may limit the significance of upcoming analyses. The reduction of theoretical uncertainties by exploiting advanced Monte Carlo (MC) event generators is the aim of this report.

\section{2. $t \bar{t} H$ modelling}

The associated production of a pair of top quarks with a Higgs boson can provide a direct measurement of the top quark Yukawa coupling. This process has not been observed yet because of its tiny signal. The latest cross section calculation at next-to-leading order (NLO) in QCD+EW for proton-proton collisions at a center-of-mass energy of $\sqrt{s}=13 \mathrm{TeV}$ has been recently published by the LHC Higgs Cross Section group [2]: $\sigma(t \bar{t} H)=0.5071_{-9.2 \%}^{+5.8 \%}($ scale) $\pm 3.6 \%$ (PDF incl. $\left.\alpha_{S}\right) \mathrm{pb}$. The PDF set considered is PDF4LHC15 and the QCD scales are set to $\mu_{0}=\mu_{R}=\mu_{F}=$ $m_{\text {top }}+m_{\mathrm{H}} / 2$, varied in the range $1 / 2<\mu / \mu_{0}<2$. An accurate modelling of the $t \bar{t} H$ process is essential to distinguish the signal from the overwhelming SM background from top quark pair production in association with jets or $W / Z$ bosons, depending on the Higgs boson decay mode. These searches are characterised by final states with a large number of jets, including heavy flavour jets, or leptons.

At present, two sets of $t \bar{t} H$ samples are available in the ATLAS experiment [3] at $\sqrt{s}=13 \mathrm{TeV}$ for Run 2 analyses [4]. The samples use inclusive NLO matrix elements interfaced to showering and hadronisation event generators. The generator employed for the hard-process is MADGraph5_AMC@NLO and events are interfaced to either Pythia8 or HERwig++. A summary of the settings and parameters used in these samples is presented in Table 1. By comparing these two $t \bar{t} H$ MC samples, the impact of the different parton shower, hadronisation and underlying event models can be evaluated. Figure 1 shows the differential cross section as function of the jet multiplicity and the transverse momentum of the $t \bar{t} H$ system for events with a semi-leptonic decay of the top quark pair and $H \rightarrow b \bar{b}$ decay mode. Selected jets are required to have $p_{T}>25 \mathrm{GeV}$ and $|\eta|<2.5$. Slightly more events with six jets (expected for the event selection applied) are observed for MADGraph5_AMC@NLO+PythIA8 prediction. In addition, the transverse momentum of the $t \bar{t} H$ system is harder for PутніA8 and the pseudo-rapidity distribution is more central. The effect is mostly visible in the lower $p_{T}$ region where a difference close to $50 \%$ is observed. Some discrepancies are also seen for the transverse momentum of the jets, $t \bar{t}$ system and Higgs boson, while good agreement is seen in the kinematic properties of the top quarks. 
Table 1: Summary of the settings in $t \bar{t} H$ MC samples used in ATLAS at $\sqrt{s}=13 \mathrm{TeV}$ [4].

\begin{tabular}{l|c|c}
\hline \hline \multicolumn{2}{c}{$t \bar{t} H, \sqrt{s}=13 \mathrm{TeV}, \mathrm{m}_{t}=172.5 \mathrm{GeV}, \mathrm{m}_{H}=125 \mathrm{GeV}$} \\
\hline ME generator & $\begin{array}{c}\text { MadGraph5_aMC@NLO } \\
+ \text { MadSpin }\end{array}$ & $\begin{array}{c}\text { MadGraph5_aMC@NLO } \\
+ \text { MadSpin }\end{array}$ \\
PS/UE generator & Herwig++ & Pythia8 \\
\hline Renormalisation and factorisation scales & $H_{T} / 2$ & $H_{T} / 2$ \\
ME \& PS/UE PDF & CT10nlo \& CTEQ6L1 & NNPDF3.0nlo \& NNPDF2.3lo \\
Tune & UE-EE-5 & A14 \\
Cross section ( \pm stat. unc.) $[\mathrm{pb}]$ & $0.447 \pm 0.007$ & $0.457 \pm 0.001$ \\
\hline \hline
\end{tabular}
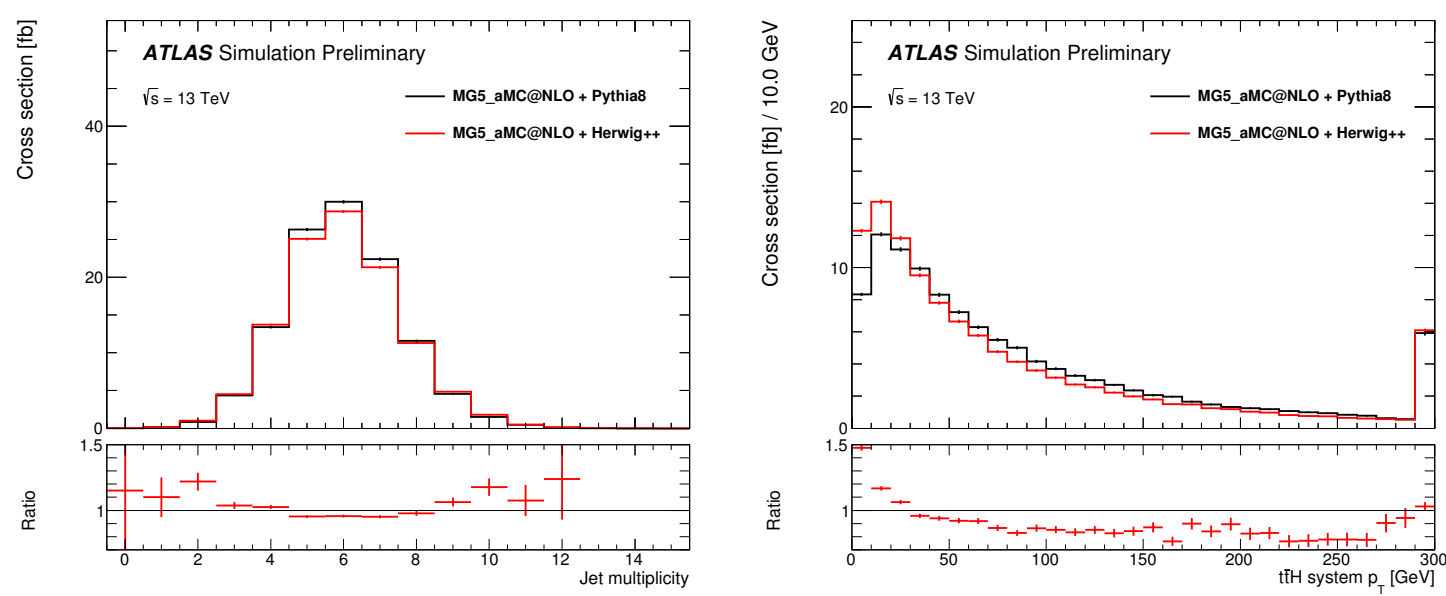

Figure 1: Cross section as a function of the number of jets in the event and the transverse momentum of the $t \bar{t} H$ system [4]. The uncertainty corresponds to the MC statistical uncertainty. The ratio in the lower pannels is taken with respect to MADGRAPH5_AMC@NLO+PyTHIA8 prediction.

\section{3. $t \bar{t}+b \bar{b}$ modelling}

The production of $t \bar{t}$ quark pairs in association with $b$-quarks constitutes a large irreducible background to the search for $t \bar{t} H$ process in the $H \rightarrow b \bar{b}$ decay channel and other searches with high number of $b$-jets in their final states. The modelling of the irreducible $t \bar{t}+b \bar{b}$ background, and the related uncertainties, plays a key role in those analyses since it is the main systematic uncertainty. The implementation of the latest theoretical developments is therefore of critical importance. It is also very challenging for the $\mathrm{MC}$ generator community. NLO predictions with massive $b$-quarks in the matrix elements, four-flavour $(4 \mathrm{~F})$ scheme, and matched to parton shower programs have become available recently within the MADGRAPH5_AMC@NLO and SHERPA+OpenLoops frameworks. Three sets of $t \bar{t}+b \bar{b}$ samples have been simulated in ATLAS at $\sqrt{s}=13 \mathrm{TeV}$ [5]. A summary of the settings and parameters used is presented in Table 2 .

In order to study additional heavy flavour production, the events are categorized based on the number of heavy flavour particle jets (which can contain one single hadron, ' $b$-jet', or double hadron jet, ' $B$-jet') that do not originate from top quark decays, with $p_{T}>15 \mathrm{GeV}$ and $|\eta|<2.5$. The " $t \bar{t}+b$ " category corresponds to an event with one resolved $b$-jet and in which the other 
$b$-jet or $B$-hadron is out of acceptance, " $t \bar{t}+b \bar{b}$ " corresponds to an event with two resolved $b$-jets, and " $t \bar{t}+B$ " is an event with one extra double hadron jet. The different $4 \mathrm{~F}$ NLO MC samples are compared to an inclusive five-flavour (5F) scheme $t \bar{t}+$ jets sample with massless $b$-quarks in the matrix elements in Figure 2. The left plot shows the absolute contribution of the various topologies. The two MadGraph5_AMC@NLO 4F NLO samples and the 5F Powheg+Pythia6 $t \bar{t}$ sample predict higher cross sections for the " $t \bar{t}+b$ " and " $t \bar{t}+b \bar{b}$ " categories than SHERPA+OPENLoops. In the right plot, the transverse momentum of the leading extra $b$-jet for the $t \bar{t}+b$ category is shown. The $4 \mathrm{~F}$ SHERPA+OpenLoops prediction is harder than the others (differences up to 20\%).

Table 2: Summary of the settings used for $t \bar{t}+b \bar{b} 4 \mathrm{~F}$ NLO samples at $\sqrt{s}=13 \mathrm{TeV}$ [5].

\begin{tabular}{|c|c|c|c|}
\hline \multicolumn{4}{|c|}{$t \bar{t}+b \bar{b}(t \bar{t} \rightarrow \mu \mu), \sqrt{s}=13 \mathrm{TeV}, \mathrm{m}_{t}=172.5 \mathrm{GeV}, \mathrm{m}_{b}=4.75 \mathrm{GeV}$} \\
\hline ME generator & MADGRAPH5_AMC@NLO & MADGRAPH5_AMC@NLO & SHERPA+OPENLOOPS \\
\hline & + MadSpin & + MadSpin & - \\
\hline $\mathrm{PS} / \mathrm{UE}$ generator & Herwig++ & Pythia8 & SHERPA \\
\hline Renormalisation scale $\mu_{R}$ & \multicolumn{3}{|c|}{$\sqrt[4]{m_{\mathrm{T}}(t) * m_{\mathrm{T}}(\bar{t}) * m_{\mathrm{T}}(b) * m_{\mathrm{T}}(\bar{b})} \quad$ (CMMPS) } \\
\hline Factorisation scale $\mu_{F}$ & \multicolumn{3}{|c|}{$H_{T} / 2$ with $H_{T}=\sum_{i \varepsilon \text { finalstate }} m_{\mathrm{T}}(i)$} \\
\hline Resummation scale $\mu_{Q}\left(Q_{s h}\right)$ & $f_{Q} \sqrt{\hat{s}}$ with & $f_{Q} \varepsilon[0.1,0.25]$ & $H_{T} / 2$ \\
\hline ME PDF & NNPDF3.0nlo $4 \mathrm{~F}$ & NNPDF3.0nlo 4F & CT10nlo $4 \mathrm{~F}$ \\
\hline PS/UE PDF & CTEQ6L1 & NNPDF2.3 & \\
\hline Tune & UE-EE-5 & A14 & author's tune \\
\hline $\mathrm{XS} \times \mathrm{BR}( \pm$ stat. unc. $)[\mathrm{pb}]$ & $0.322 \pm 0.020$ & $0.320 \pm 0.020$ & $0.315 \pm 0.020$ \\
\hline
\end{tabular}
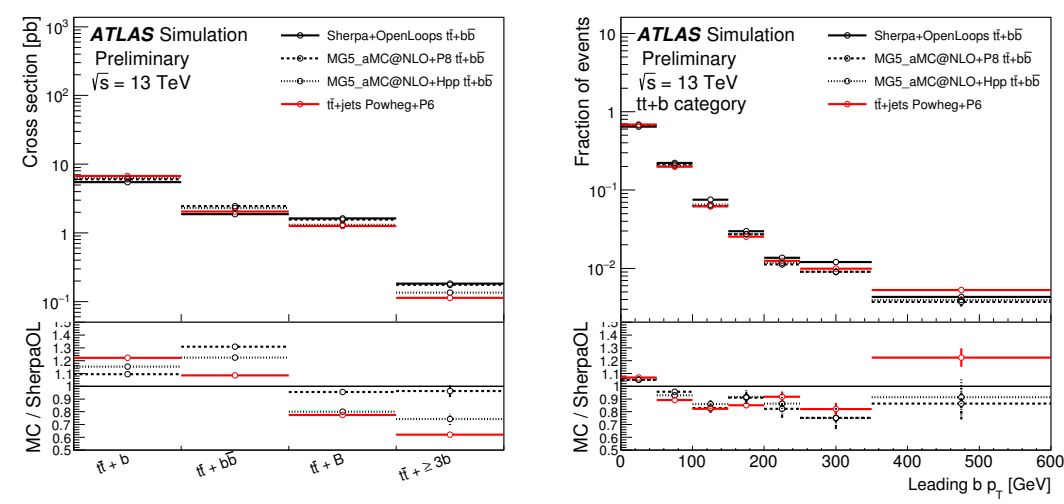

Figure 2: Different categories of $t \bar{t}+b \bar{b}$ events and transverse momentum of the leading extra $b$-jet for " $t \bar{t}+b$ " topology [5]. The uncertainties shown reflect the finite sample statistics. The ratio in the lower pannels is taken with respect to SHERPA+OpEnLOOPs prediction.

\section{References}

[1] ATLAS Collaboration, ATLAS-CONF-2016-080, https://cds.cern.ch/record/2206255 (2016).

[2] D. de Florian et al., arXiv:hep-ph/1610.07922

[3] ATLAS Collaboration, FINST 3 S08003 (2008).

[4] ATLAS Collaboration, ATL-PHYS-PUB-2016-005, https://cds.cern.ch/record/2120826 (2016).

[5] ATLAS Collaboration, ATL-PHYS-PUB-2016-016, https://cds.cern.ch/record/2205262 (2016). 